\title{
PRODUCCIÓN Y CALIDAD EN VARIEDADES DE CAFÉ (Coffea arabica L.) EN VERACRUZ, MÉXICO
}

\author{
YIELD AND QUALITY OF COFFEE CULTIVARS (Coffea arabica L.) \\ IN VERACRUZ, MÉXICO
}

\author{
Francisco Javier López-García', Esteban Escamilla-Prado', \\ Alfredo Zamarripa-Colmenero ${ }^{2}$ y J. Guillermo Cruz-Castillo ${ }^{\prime}$ *
}

\begin{abstract}
'Centro Regional Universitario Oriente, Universidad Autónoma Chapingo. Km 6 Carretera Huatusco-Xalapa. 94100, Huatusco, Veracruz; México. ${ }^{2}$ Campo Experimental Rosario Izapa, Instituto Nacional de Investigaciones Forestales, Agrícolas y Pecuarias. Km 18 Carretera Tapachula a Cacahoatan, 30870. Tuxtla Chico, Chiapas, Mexico.
\end{abstract}

*Autor de correspondencia (jcruzcastillo@yahoo.com)

\section{RESUMEN}

En Veracruz, México, el cultivo de café (Coffea arabica L.) se hace predominantemente con sistema de producción bajo sombra. Las principales variedades son Typica, Bourbon y Caturra, y la producción promedio de frutos por planta es baja en comparación con la de otros países. Aquí se evaluó la producción de fruta (café cereza), el rendimiento agroindustrial cereza-pergamino y la calidad sensorial de la bebida en 20 cultivares de café durante cinco ciclos de producción (1998 a 2003). Las variedades con mayor producción promedio de fruto (café cereza) a través de esos cinco años fueron: Catuai Amarillo (23.8 kg/planta), Caturra Rojo (22.6 kg/planta), Colombia Brote Café (23.2 kg/planta) y Colombia Brote Verde $(22.5 \mathrm{~kg} /$ planta). La variedad con mayor rendimiento agroindustrial cereza-pergamino fue Pluma Hidalgo 177 con $237.3 \mathrm{~kg}$. El rendimiento de pergamino-oro fue mejor para Colombia Brote Verde $(54.7 \mathrm{~kg})$ en comparación con Garnica Tres Cruces Porte Alto $(59.3 \mathrm{~kg})$. La proporción de granos planchuela fue alta para Caturra Amarillo y Blue Mountain, con 86.2 y $83.2 \%$ respectivamente. Pacamara tuvo la menor producción de fruto, pero presentó la mayor proporción de granos para preparación europea; este cultivar también presentó los mejores atributos en aroma, acidez y cuerpo. Para una preparación de café tipo Americano, las variedades Bourbon Salvadoreño y Bourbon Tres Cruces, tuvieron el tamaño de grano adecuado.

Palabras clave: Coffea arabica, variedades, productividad y calidad, evaluación sensorial del café.

\section{SUMMARY}

In Veracruz, México coffee (Coffea arabica L.) plantations are mainly grown under the shaded production system. The varieties typically used are Typica, Bourbon and Caturra, and their average fruit yield per plant is low compared to other countries. Fruit yield (cherry coffee), agro-industrial yield of cherryparchment coffee, and sensorial quality of the beverage were evaluated in 20 coffee cultivars growing in Veracruz, México, during five years of production (1998 to 2003). Varieties with higher average fruit yield (cherry coffee) during the evalulation period were: Catuai Amarillo (23.8 kg/plant), Caturra Rojo (22.6 kg/plant), Colombia Brote Café $(23.2 \mathrm{~kg} /$ plant) and Colombia Brote Verde $(22.5 \mathrm{~kg} / \mathrm{plant})$. The variety with the highest agro-industrial yield cherry-parchment was Pluma Hidalgo 177 with $237.3 \mathrm{~kg}$. Colombia Brote Verde produced more parchment-green coffee $(54.7 \mathrm{~kg})$ compared to Garnica Tres Cruces Porte Alto $(59.3 \mathrm{~kg})$. The largest proportion of normal grains corresponded to Caturra Amarillo and Blue Mountain, with 86.2 and $83.2 \%$ respectively. Cv. Pacamara had lower fruit yield but presented the highest proportion of grains for preparing European-style coffee; this cultivar also achieved the best attributes in smell, acidity and body. For the preparation of American-style coffee, varieties Bourbon Salvadoreño and Bourbon Tres Cruces achieved adequate grain size.

Index words: Coffea arabica, cultivars, yield and quality, sensorial evaluation of coffee.

\section{INTRODUCCIÓN}

El cafeto (Coffea arabica L.) es uno de los principales cultivos industriales en México. En los negocios de exportación los granos de café son muy apreciados (EspinosaSolares et al., 2005), y después del petróleo, es el producto más importante de exportación (DaMatta et al., 2007). En México, el cafeto se cultiva en las regiones que poseen el clima adecuado para su crecimiento, y casi toda la producción es llevada a cabo por productores con pequeñas parcelas y escaso capital para invertir en su cultivo y manejo (Calo y Wise, 2005), a diferencia de otros productores en Guatemala y Costa Rica con grandes extensiones de tierra que invierten sin escatimar para elevar la producción (Bertrand et al., 2006). En los mercados internacionales se reconocen algunos factores ambientales que influyen en la calidad del café como la altitud de la plantación (Bertrand et al., 2006), y la sombra (DaMatta, 2007), pero la variedad del cafeto también juega un papel muy importante en cualquier sistema de producción, pues del genotipo y su adaptación al ambiente depende la cantidad y la calidad de frutos a cosechar (Hein y Gatzweiler, 2005; Kathurima et al., 2009).

La mayoría de las variedades de Coffea arabica en el mundo son parecidas genéticamente, mientras que morfológicamente, presentan diferencias notables y sus frutos contrastan en calidad en pre y postcosecha (Steiger et al., 2002). En Brasil, los principales cultivares son Mundo Novo, Catuai Vermelho, y Bourbon (Perrone et al., 2008). En México, se cultivan mayormente los cultivares Typica, 
Bourbon, Caturra Rojo, Mundo Novo, Garnica y Caturra Amarillo, cuya producción promedio de frutos frescos de café (cereza) es inferior a siete sacos de café oro (menor a $2.0 \mathrm{t} \mathrm{ha}^{-1}$ de café cereza, equivalente a $8 \mathrm{qq} \mathrm{ha}^{-1}$ ), que son las unidades utilizadas en el comercio internacional de este producto (ASERCA, 2010; Escamilla et al., 2005; Varangis et al., 2002).

Específicamente, en el estado de Veracruz las vars. Typica, Garnica, Bourbon, y Caturra se cultivan ampliamente (Hernández-Solabac et al., 2011: Läderach et al., 2010). Este estado aporta $21.25 \%$ de la producción total de café cereza en México y presenta un rendimiento promedio de $2.2 \mathrm{t} \mathrm{ha}^{-1}$. El principal periodo de cosecha de frutos de café en México es de enero a marzo (ASERCA, 2010). En países como Costa Rica (Samper, 1999), Colombia y Brasil (Varangis et al., 2002) la producción promedio por hectárea es de $31.4,18$ y 17 sacos café oro ha-1 ${ }^{-1}$, respectivamente. Un saco de café oro equivale a $46 \mathrm{~kg}$ de granos de café manufacturados y previos al tostado (Espinosa-Solares et al., 2005).

Estudios sobre las características productivas y de calidad en taza de diversas variedades de café se han llevado a cabo en México, pero pocos estudios han sido publicados (Pérez-Portilla et al., 2011), y mayormente han sido limitados a uno o dos años de producción y con variedades que en un futuro tendrán menos uso por el ataque de Hemileia vastratix Berk \& Br. (Avelino et al., 2015). En otros países la evaluación agronómica de variedades de cafeto ha sido fundamental para elevar la producción y calidad en taza (Bertrand et al., 2006). Por tanto, este es un aspecto primordial que debe llevarse a cabo para mejorar el desarrollo de este cultivo en México.

Los objetivos de este estudio fueron determinar el ren- dimiento de café cereza (kg/planta) durante cinco años subsecuentes, de la calidad física del grano (medida en por ciento de frutos vanos, y en rendimiento industrial) y de la calidad en taza (evaluación sensorial) durante dos años subsecuentes, de 20 variedades de $C$. arabica cultivadas en una de las principales regiones cafetaleras del estado de Veracruz.

\section{MATERIALES Y MÉTODOS}

En 1997 se establecieron 20 variedades de Coffea arabica L. (10 de porte alto y 10 de porte bajo) con edad de 12 meses en el Municipio de Zentla, Veracruz, a 19 02' 30" $\mathrm{N}, 96^{\circ} 47^{\prime} 30^{\prime \prime} \mathrm{O}$ a una altitud promedio de $800 \mathrm{~m}$ (Cuadro 1). La distancia de plantación, para las variedades de porte bajo fue de $1.5 \times 2.0 \mathrm{~m}$, y para las variedades de porte alto fue de $2.0 \times 2.5 \mathrm{~m}$, entre plantas y entre filas, respectivamente. Los tratamientos se distribuyeron conforme a un diseño experimental de bloques completos al azar (transversales a la pendiente de $4 \%$ del terreno), con seis repeticiones y una unidad experimental de seis plantas.

La sombra de las parcelas se estableció con árboles del género Inga, 30 d después de la plantación de los cafetos, en arreglo de marco rectangular de $8 \times 6 \mathrm{~m}$. En el primer año de plantación se hicieron dos aplicaciones de fertilizante químico, con $50 \mathrm{~g}$ de urea (46N-00P-00K) por árbol, una en marzo y la otra en septiembre. En el segundo año se aplicaron $100 \mathrm{~g}$ de $18 \mathrm{~N}-46 \mathrm{P}-00 \mathrm{~K}$ en dos fechas y una encalada con $1000 \mathrm{~g}$ de cal dolomítica (Ca y Mg) por árbol de cafeto, debido a que el pH del suelo era de 4.6. En el tercer año se realizaron otras dos aplicaciones, esta vez con dosis de $200 \mathrm{~g}$ por árbol, para repetir la misma fórmula en los siguientes años evaluados. El control de la maleza se hizo de forma manual y los cafetos nunca fueron podados.

Cuadro 1: Variedades de Coffea arabica de portes bajo y alto evaluadas en Zentla, Veracruz.

\begin{tabular}{|c|c|}
\hline Porte bajo & Porte alto \\
\hline 1. Caturra Rojo & 11. Typica 947 \\
\hline 2. Catuai Amarillo & 12. Bourbon Salvadoreño \\
\hline 3. Caturra Amarillo & 13. Batie \\
\hline 4. Pacamara & 14. Blue Mountain \\
\hline 5. Garnica $F_{5}$ & 15. Typica Xhantocarpa \\
\hline 6. Garnica Iquimite & 16. Pluma Hidalgo 177 \\
\hline 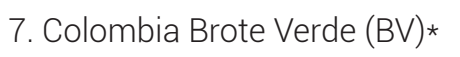 & 17. Dessie \\
\hline 8. Colombia Brote Café $(\mathrm{BC})^{\dagger}$ & 18. Garnica (TCPA)§ \\
\hline 9. Garnica (TCPB)§ & 19. Typica Tres Cruces (TC)\& \\
\hline 10. Oro Azteca & 20. Bourbon Tres Cruces $(T C)^{\S}$ \\
\hline
\end{tabular}

*Brote terminal con hojas verdes; ${ }^{\circ B}$ Brote terminal con hojas cafés; §Con tres ramas plagiotrópicas por nudo, en lugar de dos. 


\section{Variables evaluadas}

En las 20 variedades de cafeto se determinó el peso de fruta fresca o producción de café cereza (PCC) (en kg/ planta) durante cinco años de producción (1998 a 2003). La cosecha se llevó a cabo entre diciembre y febrero en cada uno de los cinco periodos de producción. La calidad física del grano y sensorial de la bebida se determinó en el ciclo 2002 a 2003. El porcentaje de frutos vanos (PFV) fue tomado en una muestra al azar de 500 frutos maduros en cada variedad, para lo cual cada muestra se depositó en una cubeta con agua y los frutos se sumergieron por 2 min, para separar los granos flotantes, los cuales se contabilizaron para obtener el porcentaje de frutos vanos (Rosas-Arellano et al., 2008).

Para cada variedad se seleccionaron al azar $6 \mathrm{~kg}$ de fruto para ser despulpados con una despulpadora de discos manual (100MEX®, México). Luego se procedió a la fermentación natural y los granos se pasaron en zarandas de madera, posteriormente el secado se hizo por exposición al sol hasta una humedad máxima de $12 \%$ determinada con un higrómetro (Delmhorst G7®, USA) conforme al método indicado por Espinosa-Solares et al. (2005). El rendimiento industrial por variedad de la conversión de café cereza a café pergamino (RCP), se obtuvo con la fórmula:

$\mathrm{RCP}=(6 \mathrm{~kg}$ cereza $\times 57.5 \mathrm{~kg}$ pergamino $) /$ peso de café resultante

En esta ecuación la cantidad $57.5 \mathrm{~kg}$ de café pergamino equivale a un quintal (1 qq) (Espinosa-Solares et al., 2005).

Para determinar el rendimiento de café pergamino a café oro (RPO), de cada muestra de café pergamino se tomaron muestras de $250 \mathrm{~g}$ los cuales se beneficiaron a café oro, se pesó el café oro resultante y se calculó el rendimiento con la ecuación de Espinosa-Solares et al. (2005):

RPO = (250 g en pergamino $\times 46 \mathrm{~kg}$ de oro $) / \mathrm{kg}$ de café en oro resultante

En donde, $46 \mathrm{~kg}$ de oro es el estándar nacional para un quintal de café oro (Espinosa-Solares et al., 2005).

Del café oro se analizaron muestras de $100 \mathrm{~g}$, en las que se separaron los granos tipo planchuela (normal), caracol, triángulo, concha y gigante (Escamilla, 1993); y los valores obtenidos se expresaron en porcentaje.

Para medir el tamaño del grano se usaron muestras de $100 \mathrm{~g}$ de café oro que se pasaron por las zarandas de los números $18,17,16,15,14$ y del $0(7.44,6.75,6.35$, $5.95,5.56$ y $0 \mathrm{~mm})$. Posteriormente se sumaron los por- centajes de los granos capturados en las zarandas 18 y 17, que son los que clasifican como preparación Europea; lo mismo se hizo con los granos de las zarandas 16 y 15 los cuales se clasifican para la preparación Americana; y por último, los granos de las zarandas 14 y 0 se clasificaron como consumo nacional. La zaranda número 18 es la que capturó los granos de mayor tamaño, y la del 0 los de menor tamaño.

La determinación de la calidad sensorial de la bebida (CSB), tras evaluar los atributos aroma, acidez, cuerpo y sabor, se llevó a cabo con un panel de cinco catadores y la escala que se utilizó varió del 1 al 15 (1 para el valor más bajo y 15 para el valor más alto). Los datos fueron capturados en el Programa Excel y se empleó el "software" Statistica 6.1 (StatSoft $\circledast$, Inc., 2003) para el análisis de varianza no paramétrico y para la comparación de medias por el método de Kruskal-Wallis (Di Rienzo et al., 2016). Los datos presentados son el promedio de dos cosechas en años consecutivos (2001 a 2003).

\section{RESULTADOS Y DISCUSIÓN}

\section{Producción de café cereza (PCC)}

En la primera cosecha, las variedades Caturras Rojo y Amarillo, Garnicas $\mathrm{F}_{5}$ Iquimite, Colombia Brote Verde (BV) y Colombia Brote Café (BC) tuvieron mayor producción de café cereza (PCC) que los otros cultivares. Catuai Amarillo y Garnica (TCPA) fueron iguales a Caturra Amarillo. Colombia Brote Café alcanzó la mayor producción en los primeros dos años. El cultivar de menor producción en los primeros dos años fue Pacamara (Cuadro 2). Al sumar la producción de cinco cosechas, los cultivares con mayor PCC fueron Catuai Amarillo, Colombia Brote Café, Caturra Rojo, Colombia Brote Verde, y fueron de porte bajo. Estos resultados coinciden con índices de producción donde los cafetos más productivos en los primeros dos años tienden a presentar mayor número de frutos en los siguientes 5 a 10 años (Walyaro y Van Der Vossen, 1979).

Las variedades Colombia con Brotes Verdes y Brotes Cafés, también denominadas Catimores, son derivadas del Híbrido de Timor $X$ Caturra y se han reportado resistentes a la roya del cafeto (Hemileia vastatrix Berk \& Br.) (Escamilla et al., 2005), lo cual las hace ventajosas para los productores. En contraste, durante este periodo la variedad con menor rendimiento $(P \leq 0.05)$ fue Pacamara de porte bajo. Otras variedades con bajos rendimientos fueron Pluma Hidalgo 177 y Blue Mountain, ambas de porte alto. Typica que es una variedad extendida en Veracruz, representada en el presente estudio por las variedades Typica 947, Typica Xhantocarpa y Typica Tres Cruces, tuvieron baja producción de fruta como ha sido indicado 
anteriormente (Escamilla, 1993). El efecto de los bloques debido a la pendiente en una dirección fue significativo ( $P$ $\leq 0.05$ ).

Ante los desafíos actuales que presenta el sector cafetalero en Veracruz, como bajo rendimiento en campo, cambio climático, infestación de roya y potencial de cafés diferenciados, es pertinente evaluar a las nuevas variedades en las diferentes regiones y bajo los sistemas de cultivo bajo sombra. Por ejemplo, la evaluación de híbridos podría ser una posibilidad, ya que en Centroamérica los híbridos de Coffea arabica han superado a las variedades tradicionales en cuanto a producción y calidad en postcosecha (Bertrand et al., 2006).

\section{Calidad física del grano}

No hubo diferencias significativas $(P \leq 0.05)$ entre las variedades en lo referente a la producción de frutos vanos (sin semilla), y los valores fueron de 3.2 a $10 \%$. La transformación de cereza a pergamino tampoco fue influenciada significativamente por el cultivar (Cuadro 3). Esto contrasta con los resultados de Espinosa-Solares et al. (2005), quienes mostraron que las vars. Typica y Mundo Novo tuvieron mayor eficiencia en conversiones de café cereza a pergamino debido al tamaño de sus frutos. En el rendimiento de pergamino a oro, Colombia Brote Verde fue más eficiente que Garnica TCPA (Cuadro 3) para conformar un quintal con menos granos (saco de $45.5 \mathrm{~kg}$ de café oro) mediante una prueba de contrastes ortogonales. Los demás cultivares fueron similares en esta variable agroindustrial (Cuadro 3).

En Costa Rica, los cultivares con un periodo de crecimiento de fruto más largo tienden a mejores rendimientos de café cereza a café oro (Alpízar, 2000); en México no se han llevado a cabo estudios al respecto. Para la investigación futura se podría considerar la evaluación en campo de híbridos de Coffea arabica que han superado a las variedades tradicionales en cuanto a producción y calidad en postcosecha. En el contexto del café mexicano con predominio de sistemas de cultivo bajo sombra y pequeños productores, son necesarios además los ensayos de variedades en varios sistemas de producción y con diversas condiciones agroecológicas.

En lo referente a las anormalidades en los granos, todas las variedades (promedio \pm error estándar) produjeron granos en forma de planchuela $(79.19 \pm 0.89)$, caracol (13.6 \pm 0.66$)$, concha $(2.06 \pm 0.20)$ y triángulo (3.69 \pm 0.52$)$, y no hubo diferencias significativas $(P \leq 0.05)$ entre las 20 variedades (Cuadro 4).

Cuadro 2. Producción de fruto en variedades de café durante cinco cosechas (1998 a 2003) en Veracruz.

\begin{tabular}{|c|c|c|c|c|c|c|}
\hline \multirow{2}{*}{ Variedad } & \multicolumn{5}{|c|}{ Cosechas (kg/planta) } & \multirow{2}{*}{ Total } \\
\hline & 1998-1999 & 1999-2000 & $2000-2001$ & $2001-2002$ & $2002-2003$ & \\
\hline Caturra Rojo & $1.1 \mathrm{a}$ & $6.7 \mathrm{~b}$ & $4.8 \mathrm{bc}$ & $6.5 \mathrm{~b}$ & $3.37 \mathrm{ef}$ & $22.6 \mathrm{ab}$ \\
\hline Catuai Amarillo & $0.9 \mathrm{bc}$ & 5.8 cde & $7.3 \mathrm{a}$ & $5.2 \mathrm{cdef}$ & $4.4 \mathrm{bcd}$ & $23.8 \mathrm{a}$ \\
\hline Caturra Amarillo & $1.0 \mathrm{ab}$ & $5.7 \mathrm{def}$ & 4.3 bcde & $6.0 \mathrm{bc}$ & $2.8 \mathrm{fgh}$ & 20.0 def \\
\hline Pacamara & $0.1 \mathrm{~g}$ & $2.8 \mathrm{i}$ & $2.7 \mathrm{~g}$ & $3.1 j$ & $3.0 \mathrm{fg}$ & $11.9 \mid$ \\
\hline Garnica $F_{5}$ & $1.0 \mathrm{ab}$ & 5.7 def & $4.5 \mathrm{bcd}$ & 4.8 efg & $2.5 \mathrm{gh}$ & 18.6 efgh \\
\hline Garnica Iquimite & $0.9 a b$ & $6.5 b c$ & 4.3 bcde & 5.7 bcde & $2.1 \mathrm{~h}$ & 19. 8def \\
\hline Colombia Brote Verde & $1.1 \mathrm{a}$ & $5.3 \mathrm{efg}$ & $5.0 \mathrm{~b}$ & $5.8 \mathrm{bcd}$ & $5.1 \mathrm{ab}$ & $22.5 a b c$ \\
\hline Colombia Brote Café & $1.1 \mathrm{a}$ & $8.2 \mathrm{a}$ & 4.0 cdef & $7.7 \mathrm{a}$ & $2.1 \mathrm{~h}$ & $23.2 a$ \\
\hline Garnica (TCPB) & $0.5 f$ & 5.2 efgh & $4.5 \mathrm{bcd}$ & 3.7 hij & $3.2 \mathrm{efg}$ & 17.2 ghij \\
\hline Oro Azteca & 0.7 cde & 5.7 cde & $3.2 \mathrm{fg}$ & 5.2 cdef & $2.1 \mathrm{~h}$ & 17.1 hij \\
\hline Typica 947 & $0.5 f$ & $5.1 \mathrm{efgh}$ & $4.6 \mathrm{bcd}$ & 3.9 ghij & 3.7 def & 17.9 fghi \\
\hline Bourbon Salvadoreño & 0.5 ef & 5.2 efgh & 3.6 defg & $4.4 \mathrm{gh}$ & $4.8 \mathrm{abc}$ & 18.8 defgh \\
\hline Batie & $0.4 f$ & 5.9 bcde & $3.3 \mathrm{efg}$ & 5.8 bcde & $4.7 \mathrm{abc}$ & 20.4 cde \\
\hline Blue Mountain & $0.4 f$ & 5.3 efgh & $3.0 \mathrm{fg}$ & $3.4 \mathrm{ij}$ & $3.6 \mathrm{def}$ & $16.0 \mathrm{ijk}$ \\
\hline Typica Xhantocarpa & $0.6 \mathrm{def}$ & 5.2 efgh & 4.0 cdef & $4.9 \mathrm{efg}$ & $5.1 \mathrm{ab}$ & $20.1 \mathrm{def}$ \\
\hline Pluma Hidalgo 177 & $0.5 f$ & $4.4 \mathrm{~h}$ & $2.7 \mathrm{~g}$ & $3.1 \mathrm{ij}$ & 4.0 cde & $14.9 \mathrm{k}$ \\
\hline Dessie & $0.4 \mathrm{f}$ & $4.9 \mathrm{fgh}$ & 3.6 defg & 4.1ghi & $4.9 \mathrm{abc}$ & 18.2 efghi \\
\hline Garnica (TCPA) & $0.8 \mathrm{bcd}$ & $6.2 \mathrm{bcd}$ & $4.7 \mathrm{bc}$ & $4.4 \mathrm{fgh}$ & $4.6 \mathrm{abcd}$ & 20.9 bcd \\
\hline Typica (TC) & $0.5 \mathrm{ef}$ & $4.7 \mathrm{gh}$ & $3.1 \mathrm{fg}$ & $3.0 \mathrm{j}$ & $3.7 \mathrm{def}$ & $15.2 \mathrm{jk}$ \\
\hline Bourbon (TC) & $0.4 \mathrm{f}$ & 5.3 efgh & 3.6 defg & $4.3 \mathrm{fgh}$ & $5.4 \mathrm{a}$ & 19.2 defg \\
\hline
\end{tabular}

Medias con letras iguales de una misma columna no son estadísticamente diferentes (Tukey, 0.05). 
Cuadro 3. Porcentaje de frutos vanos y rendimientos industriales $(\mathrm{kg})$ de 20 variedades de cafeto en Veracruz (2002 a 2003).

\begin{tabular}{|c|c|c|c|}
\hline Variedad & Fruto vano & Cereza a pergamino & Pergamino a oro \\
\hline Caturra Rojo & $4.8 \mathrm{a}$ & $238.7 \mathrm{a}$ & $55.4 \mathrm{ab}$ \\
\hline Catuai Amarillo & $3.2 \mathrm{a}$ & $244.5 \mathrm{a}$ & $55.8 \mathrm{ab}$ \\
\hline Caturra Amarillo & $5.9 \mathrm{a}$ & $252.2 \mathrm{a}$ & $56.2 \mathrm{ab}$ \\
\hline Pacamara & $7.4 \mathrm{a}$ & $239.6 \mathrm{a}$ & $56.4 \mathrm{ab}$ \\
\hline Garnica $F_{5}$ & $3.7 \mathrm{a}$ & $244.1 \mathrm{a}$ & $56.2 \mathrm{ab}$ \\
\hline Garnica Iquimite & $5.9 \mathrm{a}$ & $263.9 \mathrm{a}$ & $57.0 \mathrm{ab}$ \\
\hline Colombia Brote Verde & $8.2 \mathrm{a}$ & $253.2 \mathrm{a}$ & $54.7 b$ \\
\hline Colombia Brote Café & $6.2 \mathrm{a}$ & $259.0 \mathrm{a}$ & $56.2 a b$ \\
\hline Garnica (TCPB) & $6.0 \mathrm{a}$ & $259.4 \mathrm{a}$ & $55.8 \mathrm{ab}$ \\
\hline Oro Azteca & $6.0 \mathrm{a}$ & $245.1 \mathrm{a}$ & $56.4 \mathrm{ab}$ \\
\hline Typica 947 & $7.0 \mathrm{a}$ & $248.0 \mathrm{a}$ & $55.5 a b$ \\
\hline Bourbon Salvadoreño & $5.5 \mathrm{a}$ & $258.8 \mathrm{a}$ & $57.5 \mathrm{ab}$ \\
\hline Batie & $10.0 \mathrm{a}$ & $252.1 \mathrm{a}$ & $56.2 \mathrm{ab}$ \\
\hline Blue Mountain & $4.3 \mathrm{a}$ & $256.6 \mathrm{a}$ & $55.7 \mathrm{ab}$ \\
\hline Typica Xhantocarpa & $4.6 \mathrm{a}$ & $240.8 \mathrm{a}$ & $56.2 \mathrm{ab}$ \\
\hline Pluma Hidalgo 177 & $4.7 \mathrm{a}$ & $237.3 \mathrm{a}$ & $56.3 \mathrm{ab}$ \\
\hline Dessie & $6.9 a$ & 239.9 a & $56.7 \mathrm{ab}$ \\
\hline Garnica (TCPA) & $7.8 \mathrm{a}$ & $271.5 \mathrm{a}$ & $59.3 \mathrm{a}$ \\
\hline Typica (TC) & $7.0 \mathrm{a}$ & $258.1 \mathrm{a}$ & $57.1 \mathrm{ab}$ \\
\hline Bourbón (TC) & $7.3 \mathrm{a}$ & $270.0 \mathrm{a}$ & $58.0 \mathrm{ab}$ \\
\hline
\end{tabular}

Medias con letras iguales de una misma columna no son estadísticamente diferentes (Tukey, 0.05).

Cuadro 4. Porcentaje de anormalidades del grano de 20 variedades de café en Veracruz en 2002 a 2003.

\begin{tabular}{lcccc}
\hline Variedad & Planchuela & Caracol & Concha & Triángulo \\
\hline Caturra Rojo & $84.2 \mathrm{a}$ & $11.1 \mathrm{a}$ & $2.0 \mathrm{a}$ & $2.5 \mathrm{a}$ \\
Catuai Amarillo & $81.2 \mathrm{a}$ & $10.8 \mathrm{a}$ & $2.3 \mathrm{a}$ & $4.2 \mathrm{a}$ \\
Caturra Amarillo & $86.2 \mathrm{a}$ & $6.8 \mathrm{a}$ & $2.4 \mathrm{a}$ & $3.8 \mathrm{a}$ \\
Pacamara & $78.8 \mathrm{a}$ & $13.7 \mathrm{a}$ & $1.4 \mathrm{a}$ & $3.3 \mathrm{a}$ \\
Garnica F & $80.2 \mathrm{a}$ & $13.2 \mathrm{a}$ & $3.6 \mathrm{a}$ & $3.4 \mathrm{a}$ \\
Garnica Iquimite & $77.4 \mathrm{a}$ & $10.9 \mathrm{a}$ & $4.0 \mathrm{a}$ & $6.0 \mathrm{a}$ \\
Colombia (BV) & $80.8 \mathrm{a}$ & $14.7 \mathrm{a}$ & $1.7 \mathrm{a}$ & $2.1 \mathrm{a}$ \\
Colombia (BC) & $78.6 \mathrm{a}$ & $11.9 \mathrm{a}$ & $2.0 \mathrm{a}$ & $3.2 \mathrm{a}$ \\
Garnica (TCPB) & $79.7 \mathrm{a}$ & $11.2 \mathrm{a}$ & $1.6 \mathrm{a}$ & $5.1 \mathrm{a}$ \\
Oro Azteca & $78.9 \mathrm{a}$ & $14.6 \mathrm{a}$ & $1.8 \mathrm{a}$ & $2.8 \mathrm{a}$ \\
Typica 947 & $79.9 \mathrm{a}$ & $11.9 \mathrm{a}$ & $1.4 \mathrm{a}$ & $4.6 \mathrm{a}$ \\
Bourbón Salvadoreño & $79.7 \mathrm{a}$ & $11.2 \mathrm{a}$ & $2.6 \mathrm{a}$ & $1.4 \mathrm{a}$ \\
Batie & $76.9 \mathrm{a}$ & $18.9 \mathrm{a}$ & $0.9 \mathrm{a}$ & $1.8 \mathrm{a}$ \\
Blue Mountain & $83.2 \mathrm{a}$ & $12.5 \mathrm{a}$ & $1.4 \mathrm{a}$ & $1.9 \mathrm{a}$ \\
Typica Xhantocarpa & $80.2 \mathrm{a}$ & $14.3 \mathrm{a}$ & $1.0 \mathrm{a}$ & $1.0 \mathrm{a}$ \\
Pluma Hidalgo 177 & $82.4 \mathrm{a}$ & $13.7 \mathrm{a}$ & $1.1 \mathrm{a}$ & $1.2 \mathrm{a}$ \\
Dessie & $76.1 \mathrm{a}$ & $18.9 \mathrm{a}$ & $1.3 \mathrm{a}$ & $3.9 \mathrm{a}$ \\
Garnica (TCPA) & $72.2 \mathrm{a}$ & $13.0 \mathrm{a}$ & $3.7 \mathrm{a}$ & $7.1 \mathrm{a}$ \\
Typica (TC) & $75.2 \mathrm{a}$ & $16.8 \mathrm{a}$ & $2.8 \mathrm{a}$ & $2.2 \mathrm{a}$ \\
Bourbón (TC) & $71.9 \mathrm{a}$ & $17.0 \mathrm{a}$ & & \\
\hline
\end{tabular}

Medias con letras iguales de una misma columna no son estadísticamente diferentes (Tukey, 0.05). 
El tamaño del grano de café es una característica determinante por los compradores al mayoreo. Los europeos prefieren los tamaños de semilla más grande, ya que la preparación Europea corresponde a la criba 17 y 18; en cambio, la preparación Americana es con las cribas 15 y 16. La var. Pacamara presentó un porcentaje de preparación Europea de hasta 89.2 en dos años consecutivos (Cuadro 5). Las variedades con menor tamaño de grano y adecuadas para la preparación Americana fueron Bourbón Salvadoreño y Bourbón Tres Cruces (Cuadro 5). Sin embargo, el tamaño de grano no ha sido relacionado significativamente con la calidad en taza (Kathurima et al., 2009). En el cafeto una mayor carga de frutos por arbusto se asocia con granos de menor tamaño (Vaast et al., 2006) y de menor peso seco (Vaast et al., 2005). En este contexto, la var. Pacamara tuvo la menor producción de frutos (11.9 $\mathrm{kg} /$ planta) en 5 años y el tamaño grande de sus frutos, determinado por su alto porcentaje de preparación Europea, lo que se puede relacionar con su genética con su alta proporción hoja:fruto por arbusto (Vaast et al., 2006), y con su baja producción de fruto por árbol.

\section{Calidad sensorial de la bebida}

Se determinaron diferencias significativas en los atributos aroma, acidez y cuerpo. El sabor fue similar $(P \leq 0.05)$ para todas las variedades (Cuadro 6) en dos cosechas en años consecutivos. Los granos de café de las vars. Pacamara, Colombia Brote Café, Colombia Brote Verde, Typica 947 y Garnica TCPA (Tres Cruces Porte Alto), presentaron mejor aroma $(P \leq 0.05)$ que Garnica TCPB (Garnica Tres Cruces Porte Bajo) (Cuadro 6). Sin embargo, la acidez fue baja para Garnica TCPB. Las variedades con más bajo cuerpo en la bebida fueron Caturra Rojo, Garnica $\mathrm{F}_{5^{\prime}}$ Garnica Iquimite y Garnica (TCPB). En general, las demás variedades fueron muy parecidas en esta característica $(\mathrm{P} \leq$ 0.05). La composición bioquímica de los granos de café está influenciada por factores genéticos (Montagnon et al., 1998). Sin embargo, la calidad en taza puede estar más relacionada con el microlima de las regiones de producción de café (Avelino et al., 2005).

Cuadro 5. Tamaño del grano de café (\%) para preparaciones Europea y Americana en dos años consecutivos (2001 a 2003).

\begin{tabular}{|c|c|c|c|c|c|c|}
\hline \multirow{2}{*}{ Variedad } & \multicolumn{2}{|c|}{ Primer año } & \multicolumn{2}{|c|}{ Segundo año } & \multicolumn{2}{|c|}{ Promedio (\%) } \\
\hline & Europea & Americana & Europea & Americana & Europea & Americana \\
\hline Caturra Rojo & $76.7 b$ & $23.2 \mathrm{~d}$ & $75.0 c$ & $25.0 \mathrm{c}$ & 75.9 bc & $24.1 \mathrm{c}$ \\
\hline Catuai Amarillo & $81.7 b$ & $18.2 \mathrm{e}$ & $81.5 b$ & $18.5 d$ & $81.6 b$ & $18.3 d$ \\
\hline Caturra Amarillo & $76.9 b$ & $23.0 \mathrm{~d}$ & $78.1 \mathrm{~b}$ & $21.9 c$ & $77.5 b$ & $22.4 \mathrm{c}$ \\
\hline Pacamara & $91.9 \mathrm{a}$ & $8.0 \mathrm{~g}$ & $87.5 \mathrm{a}$ & $13.5 \mathrm{e}$ & $89.2 a$ & $10.7 \mathrm{e}$ \\
\hline Garnica $\mathrm{F}_{5}$ & $74.5 b$ & $25.4 \mathrm{c}$ & $84.2 \mathrm{ab}$ & 15.8 e & $79.3 b$ & $20.6 \mathrm{~cd}$ \\
\hline Garnica Iquimite & $77.9 \mathrm{~b}$ & $22.0 \mathrm{~d}$ & $70.5 d$ & $29.5 b$ & $74.2 \mathrm{bc}$ & $25.7 \mathrm{bc}$ \\
\hline Colombia (BV) & $75.4 b$ & $24.5 \mathrm{c}$ & $77.5 \mathrm{bc}$ & $22.5 \mathrm{c}$ & $76.5 \mathrm{bc}$ & $23.5 c$ \\
\hline Colombia (BC) & $81.9 b$ & $18.0 \mathrm{e}$ & $82.0 \mathrm{~b}$ & $18.0 \mathrm{~d}$ & $81.9 b$ & $18.0 \mathrm{~d}$ \\
\hline Garnica (TCPB) & $87.1 \mathrm{ab}$ & $12.8 f$ & $65.0 \mathrm{e}$ & $35.0 \mathrm{a}$ & $76.0 \mathrm{bc}$ & $23.9 \mathrm{c}$ \\
\hline Oro Azteca & $72.2 \mathrm{c}$ & $27.7 c$ & $72.0 \mathrm{~d}$ & $28.0 \mathrm{~b}$ & $72.1 \mathrm{c}$ & $27.8 b$ \\
\hline Typica 947 & $73.7 b$ & $26.2 \mathrm{c}$ & $83.0 \mathrm{~b}$ & $17.0 \mathrm{~d}$ & $78.3 b$ & $21.6 c$ \\
\hline Bourbón Salvadoreño & $56.6 \mathrm{~d}$ & $43.3 \mathrm{a}$ & $72.7 \mathrm{~d}$ & $27.3 b$ & $64.6 \mathrm{~d}$ & $35.3 \mathrm{a}$ \\
\hline Batie & $80.1 \mathrm{~b}$ & $19.8 \mathrm{e}$ & $74.6 \mathrm{c}$ & $25.4 \mathrm{c}$ & $77.3 \mathrm{bc}$ & $22.6 \mathrm{c}$ \\
\hline Blue Mountain & $72.7 \mathrm{c}$ & $27.2 \mathrm{c}$ & $79.3 \mathrm{bc}$ & $20.7 \mathrm{~cd}$ & $76.0 \mathrm{bc}$ & $23.9 \mathrm{c}$ \\
\hline Typica Xhantocarpa & $75.6 b$ & $24.3 d$ & 67.3 de & $32.7 \mathrm{ab}$ & $71.4 \mathrm{c}$ & $28.5 b$ \\
\hline Pluma Hidalgo 177 & $79.8 b$ & $20.1 \mathrm{e}$ & $75.7 \mathrm{c}$ & $24.3 \mathrm{c}$ & $77.7 \mathrm{bc}$ & $22.2 \mathrm{c}$ \\
\hline Dessie & $76.1 \mathrm{~b}$ & $23.8 \mathrm{~d}$ & $82.0 \mathrm{~b}$ & $18.0 \mathrm{~d}$ & $79.0 b$ & $20.9 \mathrm{~cd}$ \\
\hline Garnica (TCPA) & $72.5 \mathrm{c}$ & $27.5 \mathrm{c}$ & $70.4 d$ & $29.6 b$ & $71.4 \mathrm{c}$ & $28.5 b$ \\
\hline Typica (TC) & $71.0 \mathrm{c}$ & $28.9 \mathrm{c}$ & $73.2 \mathrm{~cd}$ & $26.8 b$ & $72.1 \mathrm{c}$ & $27.8 b$ \\
\hline Bourbon (TC) & $61.5 \mathrm{~d}$ & $38.4 b$ & $64.7 \mathrm{e}$ & $35.3 a$ & $63.1 \mathrm{~d}$ & $36.8 \mathrm{a}$ \\
\hline
\end{tabular}

Medias con letras iguales de una misma columna no son estadísticamente diferentes (Tukey, 0.05). 
Cuadro 6. Evaluación sensorial en taza para determinar aroma, acidez, sabor y cuerpo para 20 variedades de cafeto en Veracruz. Los valores son un promedio de dos cosechas en años consecutivos (2001 a 2003). En la escala, 1 es el valor más bajo y 15 el más alto.

\begin{tabular}{|c|c|c|c|c|}
\hline Variedad & Aroma & Acidez & Sabor & Cuerpo \\
\hline Caturra Rojo & $10.2 \mathrm{bc}$ & $9.6 \mathrm{bcd}$ & $6.7 \mathrm{a}$ & $7.6 \mathrm{~b}$ \\
\hline Catuai Amarillo & $10.2 \mathrm{bc}$ & $9.5 \mathrm{~cd}$ & $6.8 \mathrm{a}$ & $7.8 \mathrm{ab}$ \\
\hline Caturra Amarillo & 10.2 bc & $9.3 \mathrm{~cd}$ & $6.5 a$ & $8.1 \mathrm{ab}$ \\
\hline Pacamara & $12.4 \mathrm{a}$ & $12.5 \mathrm{a}$ & $6.0 \mathrm{a}$ & $9.7 \mathrm{a}$ \\
\hline Garnica $F_{5}$ & 10.4 bc & $9.6 \mathrm{bcd}$ & $6.5 \mathrm{a}$ & $7.7 b$ \\
\hline Garnica Iquimite & $10.2 b c$ & $9.3 \mathrm{~cd}$ & $6.7 \mathrm{a}$ & $7.6 \mathrm{~b}$ \\
\hline Colombia (BV) & $10.9 a b$ & $10.3 \mathrm{abcd}$ & $6.1 \mathrm{a}$ & $8.6 a b$ \\
\hline Colombia (BC) & $11.5 \mathrm{ab}$ & $11.8 \mathrm{ab}$ & $6.2 \mathrm{a}$ & $9.0 \mathrm{ab}$ \\
\hline Garnica (TCPB) & $9.8 \mathrm{c}$ & $8.8 \mathrm{~d}$ & $6.8 \mathrm{a}$ & $7.4 \mathrm{~b}$ \\
\hline Oro Azteca & $11.2 \mathrm{abc}$ & $10.3 \mathrm{abcd}$ & $6.5 a$ & $8.2 a b$ \\
\hline Typica 947 & $11.5 \mathrm{ab}$ & $10.3 \mathrm{abcd}$ & $6.4 \mathrm{a}$ & $8.0 \mathrm{ab}$ \\
\hline Bourbón Salvadoreño & $10.6 \mathrm{bc}$ & 9.8 bcd & $6.4 \mathrm{a}$ & $7.8 \mathrm{ab}$ \\
\hline Batie & $10.8 \mathrm{abc}$ & $10.7 \mathrm{abcd}$ & $6.2 \mathrm{a}$ & $8.6 a b$ \\
\hline Blue Mountain & $11.2 \mathrm{abc}$ & $10.7 \mathrm{abcd}$ & $6.1 \mathrm{a}$ & $8.6 a b$ \\
\hline Typica Xhantocarpa & $11.4 \mathrm{abc}$ & $10.4 \mathrm{abcd}$ & $6.5 a$ & $8.3 a b$ \\
\hline Pluma Hidalgo 177 & $11.3 \mathrm{abc}$ & $10.8 \mathrm{abcd}$ & $6.3 \mathrm{a}$ & $8.7 \mathrm{ab}$ \\
\hline Dessie & $11.1 \mathrm{abc}$ & $10.8 \mathrm{abcd}$ & $6.1 \mathrm{a}$ & $8.7 \mathrm{ab}$ \\
\hline Garnica (TCPA) & $11.5 \mathrm{ab}$ & $11.5 \mathrm{abc}$ & $6.0 \mathrm{a}$ & $9.1 \mathrm{ab}$ \\
\hline Typica (TC) & $11.1 \mathrm{abc}$ & $10.4 \mathrm{abcd}$ & $6.4 \mathrm{a}$ & $8.2 a b$ \\
\hline Bourbón (TC) & $11.2 \mathrm{abc}$ & $10.7 \mathrm{abcd}$ & $6.5 a$ & $8.7 \mathrm{ab}$ \\
\hline
\end{tabular}

Medias con letras iguales de una misma columna no son estadísticamente diferentes (Kruskal-Wallis, 0.05).

\section{CONCLUSIONES}

Las variedades de porte bajo Catuai Amarillo, Colombia Brote Café, Caturra Rojo y Colombia Brote Verde mostraron alta productividad por planta con un acumulado en cinco años consecutivos por arriba de los $20 \mathrm{~kg} /$ planta, lo que supera a todas las variedades de porte alto. En cuanto a la calidad física del grano, para las variables planchuela, caracol, concha y triángulo no hubo diferencias significativas entre variedades. El cv. Pacamara tuvo baja producción de grano; sin embargo, presentó la mayor proporción de granos para preparación Europea y los mejores atributos en la calidad sensorial en aroma, acidez y cuerpo. Las vars. Bourbon Salvadoreño y Bourbon Tres Cruces tuvieron un tamaño de grano ideal para una preparación tipo Americana.

\section{BIBLIOGRAFÍA}

Alpízar J. M. (2000) Comportamiento de los rendimientos de beneficiado de café de Costa Rica por zonas de producción, según registros de 15 años. In: L. Zamora y J. Echeverri (eds.). XIX Simposio Latinoamericano de Caficultura, Memoria, ICAFE San
José, Costa Rica. pp:467-471.

ASERCA, Agencia de Servicios a la Comercialización y Desarrollo de Mercados Agropecuarias (2010) Escenario actual del café. SAGARPA. 16 p. http://www.aserca.gob.mx/Paginas/default.aspx.

Avelino J., B. Barboza, J. C. Araya, C. Fonseca, F. Davrieux, B. Guyot and C. Cilas (2005) Effects of slope exposure, altitude and yield on coffee quality in two altitude terroirs of Costa Rica, Orosi and Santa Maria de Dota. Journal of the Science of Food and Agriculture 85:1869-1876.

Avelino J., M. Cristancho, S. Georgiou, P. Imbach, L. Aguilar, G. Bomemann, P. Läderach, F. Anzueto, A.J. Hruska and C. Morales (2015) The coffee rust crises in Colombia and Central America (2008-2013): impacts, plausible causes and proposed solution. Food Security 7:313-321.

Bertrand B., P. Vaast, E. Alpizar, H. Etienne, F. Davrieux and P. Charmetant (2006) Comparison of bean biochemical composition and beverage quality of Arabica hybrids involving Sudanese-Ethiopian origins with traditional varieties at various elevations in Central America. Tree Physiology 26:1239-1248.

Calo M. and T. A. Wise (2005) Revaluing Peasant Coffee Production: Organic and Fair Trade Markets in Mexico. Global Development and Environment Institute. Tufts University. 57 p.

DaMatta F. M., P. C. Ronchi, M. Maestri and S. R. Barros (2007) Ecophysiology of coffee growth and production. Brazilian Journal of Plant Physiology 19:485-510

Di Rienzo J. A., F. Casanoves, M. G. Balzarini, L. González, M. Tablada y C. W. Robledo (2016) InfoStat versión 2016. Grupo InfoStat, FCA, Universidad Nacional de Córdoba, Argentina. 
Escamilla P. E. (1993) El Café Cereza en México Tecnología de la Producción. Universidad Autónoma Chapingo. 116 p.

Escamilla E., O. Ruiz, G. Díaz, C. Landeros, D.E. Platas, A. Zamarripa y V. A. González (2005) El agroecosistema café orgánico en México. Manejo Integrado de Plagas y Agroecología 76:5-16.

Espinosa-Solares T., J. G. Cruz-Castillo, O. A. Montesinos-López and A. Hernández-Montes (2005) Raw coffee processing yield affected more by cultivar than by harvest date. The Journal of Agriculture of the University of Puerto Rico 89:169-180.

Hein L. and F. Gatzweiler (2005) The economic value of coffee (Coffea arabica) genetic resources. Ecological Economics 60:176-185.

Hernández-Solabac J. A. M., M. E. Nava-Tablada, S. Díaz-Cárdenas, E. Pérez-Portilla y E. Escamilla-Prado (2011) Migración internacional y manejo del café en dos comunidades del Centro de Veracruz. Tropical and Subtropical Agroecosystems 14:807-818.

Kathurima C. W., B. M. Gichimu, G. M. Kenji, S. M. Muhoho and R. Boulanger (2009) Evaluation of beverage quality and green bean physical characteristics of selected Arabica coffee genotypes in Kenya. African Journal of Food Science 3:365-371.

Läderach P., T. Oberthür, S. Cook, M. Estrada-Iza, J. A. Pohlan, M. Fisher and R. Rosales-Lechuga (2010) Systematic agronomic farm management for improved coffee quality. Field Crops Research 120:321-329.

Montagnon C., B. Guyot, C. Cilas and T. Leroy (1998) Genetic parameters of several biochemical compounds from green coffee, Coffea canephora. Plant Breeding 117:576-578.

Pérez-Portilla E., S. Bonilla-Cruz, J. A. M. Hernández-Solabac y J. G. PartidaSedas (2011) Estrategia de mejoramiento de la producción cafetalera de la organización Campesinos Ecológicos de la Sierra Madre de Chiapas: caracterización de la bebida de café. Revista de Geografía Agrícola 46-47:7-18.
Perrone D., A. Farah, C. M. Donangelo, T. de Paulis and P. R. Martin (2008) Comprehensive analysis of major and minor chlorogenic acids and lactones in economically relevant Brazilian coffee cultivars Food Chemistry 106:859-867.

Rosas-Arellano J., E. Escamilla-Prado y 0. Ruiz-Rosado (2008) Relación de los nutrimentos del suelo con las características físicas y sensoriales del café orgánico. Terra Latinoamericana 26:375-384.

Samper K. M. (1999) Trayectoria y viabilidad de la Caficultura Centroamericanas. In: B. Bertrand y B. Rapidel (eds). Desafíos de la Caficultura en Centroamérica. IICA. PROMECAFE. CIRAD. IRD. CCCR. Francia. Costa Rica. pp:1-68.

StatSoft Inc. (2003) Statistica. Data Análisis Software System. Version 6. In:www.statsoft.com

Steiger D. L., C. Nagai, P. H. Moore, C. W. Morden, R. V. Osgood and R. Ming (2002) AFLP analysis of genetic diversity within and among Coffea arabica cultivars. Theoretical and Applied Genetics 105:209-215

Vaast P., J. Angrand, N. Franck, J. Dauzat and M. Génard (2005) Fruit load and branch ring-barking affect carbon allocation and photosynthesis of leaf and fruit of Coffea arabica in the field. Tree Physiology 25:753-760.

Vaast P., B. Bertrand, J.-J. Perriot, B. Guyot and M. Génard (2006) Fruit thinning and shade improve bean characteristics and beverage quality of coffee (Coffea arabica L.) under optimal conditions. Journal of the Science of Food and Agriculture 86:197-204.

Varangis P., P. Siegel, D. Giovanucci y B. Lewin (2002) Región Centroamericana. La Crisis Cafetalera: Efectos y Estrategias para Hacerle Frente. World Bank Policy Research Working Paper. Informe 2993. pp:11-12. https://books.google.com.mx/ books?id=EevOmALwN5gC

Walyaro D. J. and H. A. M. Van Der Vossen (1979) Early determination of yield potential in Arabica coffe by applying index selection. Euphytica 28:565-472 